predictor to detect patients with urinary uric acid underexcretion, who could then be treated with uricosuric drugs.

Disclosure of Interest: None declared

DOI: 10.1136/annrheumdis-2018-eular.5005

\section{FRI0249 AUDIT OF THE MANAGEMENT OF GOUT -ARE WE DOING IT RIGHT?}

T.J. Khan, S. Kamath, E. Roddy. Rheumatology, Haywood Hospital, Stoke-OnTrent, UK

Background: Gout is the most prevalent inflammatory arthritis, affecting $2.5 \%$ of adults in the UK. However, management is often inadequate in both primary and secondary care, with only $45 \%$ of patients achieving target serum urate (SUA) level $<360 \mu \mathrm{mol} / \mathrm{L}$ over 12 months in UK rheumatology clinics. A better understanding of how well gout is managed in different areas of our service (discharge to GP, general rheumatology follow-up clinics, and specialist gout clinic) will inform service redesign.

Objectives: To compare the management of gout in the rheumatology service against the 2007 British Society for Rheumatology (BSR) and 2006 European League Against Rheumatism (EULAR) gout guidelines, and the NICE febuxostat technology appraisal (TA164).

Methods: We retrospectively audited all new out-patient referrals with gout seen in our department over a 12 month period (January-December 2015). Data were collected by electronic review of case notes and completion of a structured proforma. Three mutually exclusive groups were compared: those seen once in rheumatology and discharged to GP (group1), followed-up in general rheumatology clinics (group2), or followed-up in a specialist gout clinic (group3). Follow-up SUA levels were specifically compared to EULAR $(<360 \mu \mathrm{mol} / \mathrm{L})$ and BSR $(<300 \mu \mathrm{mol} /$ L) treatment targets.

Results: 150 new consecutive gout referrals (50 per group) were included in the audit: $83 \%$ were male and mean age was 62 years. Gout was diagnosed by monosodium urate crystal identification in $16(11 \%)$ and $25 \%$ had tophi. $43(29 \%)$ patients were already on ULT, and 107 (71\%) patients were newly commenced on ULT. Prophylactic medications were co-prescribed in $86 \%$ (130) cases. 44 patients were taking diuretics; diuretics were advised to be stopped or reduced in $12 \%$ cases. Nearly all patients starting allopurinol commenced a daily dose of $\leq 100 \mathrm{mg}$ (99\% cases). Of the patients started on a uricosuric/febuxostat, $92 \%$ had taken allopurinol previously. Of the 15 patients commenced on febuxostat, 3 $(15 \%)$ had ischaemic heart disease (IHD) or cardiac failure. Chronic kidney disease (CKD) stage 3 (group1 28\%, group2 22\%, group3 34\%), CKD stage 4/5 (2\%, $2 \%, 10 \%)$, IHD/cardiac failure $(30 \%, 30 \%, 40 \%)$, clinically evident tophi $(16 \%$, $18 \%, 42 \%)$ and previous allopurinol intolerance $(10 \%, 14 \%, 24 \%)$ were more common in group 3 than groups 1 and 2 . After 12 months, only $90(60 \%)$ patients achieved target $<360 \mu \mathrm{mol} / \mathrm{L}$ (group1 $42 \%$, group2 64\%, group3 62\%) and 47 (31\%) patients achieved target SUA $<300 \mu \mathrm{mol} / \mathrm{L}$ (group1 20\%, group2 34\%, group3 36\%)

Conclusions: Allopurinol starting dose, use of prophylaxis, and use of allopurinol first-line concorded well with national and international guidelines. We achieved target SUA levels more commonly than the UK national average in a recent national rheumatology audit. Patients discharged to the GP with a management plan prior to achieving a target SUA level achieved target less frequently suggesting that rheumatologists should follow patients in order to ensure treatment is escalated until the target SUA level is achieved.

Disclosure of Interest: None declared

DOI: 10.1136/annrheumdis-2018-eular.4622

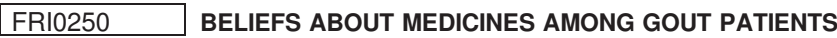 - DATA FROM THE NOR-GOUT STUDY}

T. Uhlig ${ }^{1}$, L.F. Karoliussen ${ }^{1}$, J. Stjärne ${ }^{2}$, E.A. Haavardsholm ${ }^{1}$, T.K. Kvien ${ }^{1}$, H. B. Hammer ${ }^{1}$ ' Rheumatology, Diakonhjemmet Hospital, Oslo; ${ }^{2}$ Rheumatology, Betanien Hospital, Skien, Norway

Background: Low adherence to medication is a concern in gout where urate lowering therapy (ULT) is indicated to prevent disease severity and comorbidities. The beliefs patients have about medication may impact on the success of achieving these treatment goals.

Objectives: To study which factors were associated to beliefs about medicines in patients with a recent gout attack and a need for ULT.

Methods: Baseline data from a prospective observational study were used in patients with crystal-proven gout who presented after a recent gout flare with insufficiently treated serum urate (sUA) level $(>360 \mu \mathrm{mol} / \mathrm{L} />6 \mathrm{mg} / \mathrm{dl}$ ). In these patients a treat-to-target approach was planned to meet the treatment target (sUA $<360 \mu \mathrm{mol} / \mathrm{L}$, or $<300 \mu \mathrm{mol} / \mathrm{L}$ if clinical tophi). Assessment included demographic and clinical data, baseline serum urate levels, medication, self-administered comorbidity questionnaire (SCQ), physical function (HAQ), and SF-36 mental (MCS) and physical component summaries.

The Beliefs in Medicines Questionnaire (BMQ) ${ }^{1}$ assesses patient beliefs about medicines on four subscales: necessity and concerns specific for the patient, and generally on overuse and harm. Respondents indicated their degree of agreement with each individual statement about medicines on a 5-point Likert scale, ( $1=$ strongly disagree to $5=$ strongly agree). Scores within the four subscales (necessity, concerns, overuse, harm) were summed (ranges 5-25 and 4-20). Calculation of the necessity-concerns differential gave the relative importance for the patient for taking medicines.

Results: 163 patients were included at baseline, $93.3 \%$ men, $90.5 \%$ caucasian, mean (SD) age 57.0 (14.1) years, disease duration 8.0 (7.7) years). Mean sUA level was 487 (SD 82) $\mu \mathrm{mol} / \mathrm{L}$ at baseline, body mass index $28.9(4.7) \mathrm{kg} / \mathrm{m} 2$, comorbidity score (SCQ) 3.6 (3.2), and physical function (HAQ) 0.35 (0.55). $18.8 \%(n=28)$ had tophi, and $30.1 \%(n=43)$ were using allopurinol.

Scores for the BMQ subscales (SD) were for necessity 16.8 (4.3), concerns 13.7 (5.0), overuse 10.6 (2.7), and harm 9.5 (2.4). The specific necessity-concerns dif ferential was 3.1 (5.7), with median 2.5. Patients expressing higher versus lower beliefs in importance of medication (necessity-concern higher than median) demonstrated in bivariate comparisons statistically significantly differences (table 1). The level of serum urate was not associated with any BMQ subscale.

In logistic regression analyses, also adjusting for age and gender in the final model, high beliefs in the relative importance of medication were independently associated with not using allopurinol medication (OR $0.41,95 \% \mathrm{Cl} 0.18-0.94$ ), with higher $\mathrm{BMI}(\mathrm{OR}=1.11$ per unit, $95 \% \mathrm{Cl} 1.02-1.20)$, and better mental health (SF36 MCS) (OR 1.04 per unit, 95\% Cl 1.01-1.08).

\begin{tabular}{lccc}
\hline & High belief (>median) & Low belief & p-value \\
\hline BMI & 29.6 & 28.2 & 0.048 \\
Comorbidity score (SCQ) & 2.3 & 4.4 & 0.02 \\
Taking allopurinol & $25.8 \%$ & $37.9 \%$ & 0.09 \\
SF36 MCS & 50.8 & 47.1 & 0.03 \\
BMQ overuse & 9.9 & 11.4 & 0.001 \\
BMQ harm & 8.9 & 10.2 & 0.001 \\
\hline
\end{tabular}

Conclusions: Unexpectedly, using allopurinol medication was inversely associated with high beliefs, whereas higher BMI and better mental health were positively associated with high beliefs in the importance of medication in gout patients. These findings do not allow conclusions on causality, and beliefs in medicines in gout patients should also be studied longitudinally and in relationship to therapy response.

\section{REFERENCE:}

[1] Horne R, et al. Psychology \& Health 1999;14(1):1-24.

Disclosure of Interest: None declared

DOI: 10.1136/annrheumdis-2018-eular.4435

\section{FRI0251 PREVALENCE OF GOUT AND THE USE OF URATE LOWERING AGENTS IN US GENERAL POPULATION NHANES 2007-2016}

T.T. Cheung, M.F. Tsoi, B.M.Y. Cheung, W.C.S. Lau. Medicine, The University of Hong Kong, Hong Kong

Background: The prevalence of gout has been increasing worldwide. Previous study using National Health and Nutritional Examination Survey (NHANES) showed an increase of $1.2 \%$ in the prevalence of gout in the US general population from 1988 to 2007 . However, it is unknown if this trend continued over the past decades. Therefore, we would like to determine the prevalence of gout in the US general population using NHANES 2007 to 2016. In addition, the use of urate lowering agents among patients with gout was analysed.

Objectives: To estimate the prevalence of gout and the use of urate lowering agents using the National Health and Nutritional Examination Survey from 2007 to 2016

Methods: Adult participants in NHANES 2007-2016 were included in the analysis. NHANES is a continuous national survey conducted by the US Centres for Disease Control and Prevention and is designed to evaluate the health and nutritional status of adults and children in the US. They are based on a representative sample of the non-institutionalised US civilian population. Each participant represents approximately 50000 Americans.

The primary outcome was self-reported gout. Factors associated with gout, such as body weight, drinking habit, history of chronic kidney disease, use of aspirin and thiazide diuretics were evaluated. Prescriptions of allopurinol, febuxostat or probenacid were retrieved from the NHANES dataset to evaluate the use of urate lowering agents among patients with gout. 
Results were analysed using SPSS complex sample module version 22. Multiple regression was used to analyse the difference in prevalence of gout and utilisation of urate lowering agents.

Results: 23482 adults participants were included in the analysis. There was no further increase in the prevalence of gout in the general US population. The prevalence was $3.82 \%$ and $3.84 \%$ in NHANES 2007-2010 and 2011-2016, respectively $(\mathrm{p}=0.966)$.

Although there is a decreasing trend in the serum urate levels among patients with gout, the utilisation of urate lowering agents remained low. Only $28.2 \%$ and $29.4 \%$ of patients with gout were prescribed urate lowering agents in 2007-2010 and 2011-2014, respectively.

Among patients with gout, history of chronic kidney disease and use of thiazide diuretics are the most significant negative predictors for achieving the therapeutic target of SUV $<6 \mathrm{mg} / \mathrm{dL}$ (Odds ratio of 0.23 and 0.41 , respectively). Use of aspirin was not a significant predictor for treatment failure.

\begin{tabular}{|c|c|c|c|c|c|c|}
\hline 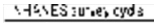 & $20: \because 0.2$ & $2 \times 0.20:$ & $30+1.012$ & 2610iviti & a) 5.200 & P,slus \\
\hline 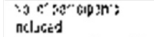 & $5 \xi^{2}$ & 8215 & sक: & 3735 & 济早 & \\
\hline 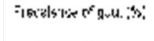 & $39.3 .4<51$ & $3 \pi[-4.1]$ & 3012.8 .6 & 4. & 3.9 .2 .4 .4 & $033 z$ \\
\hline 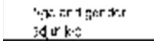 & $343: 24$ & | & $\because \div 13 \cdot \sqrt{3}$ & 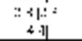 & 1 & \\
\hline
\end{tabular}

\begin{tabular}{|c|c|c|c|c|c|}
\hline & 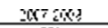 & $2003 \times 10$ & $201150 ?$ & $2 \times 132014$ & Powi: \\
\hline 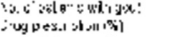 & 259 & 23 & 239 & $\{36$ & \\
\hline 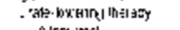 & $2^{\prime} \ell(i 1(-5+0)$ & $35819-92$ & 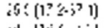 & $30 ;|243-60|$ & $x \leqslant 0$ \\
\hline s.lept. nci & 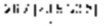 & 1 & 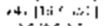 & אים & W 'lis \\
\hline 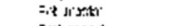 & $\therefore$ & 0 & $a c n=25$ & 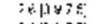 & 0.030 \\
\hline$=\infty \mathrm{n}$ r:es & 80.513 & $1.203 \%$. & $0=0.2 .1^{\circ}$ & $: 210.3 z$ & 0.179 \\
\hline 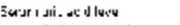 & a $72 a 0.99$ & $5.63 \pm 0 \div$ & $3.0 \pm 0.24$ & $(\varepsilon)=3 \cdot 1$ & - 060 \\
\hline 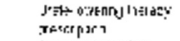 & , N\&n. & $\because 2 \pm 0$ 's &, $0.9 \pm 0 .: i$ & $6,3= \pm i$ & $\cdot .0 .1$ \\
\hline 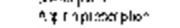 & $5 \div .06$ & :27.041 & $\$+1,0 \leq=$ & ( $\leqslant 8-142$ & ut.s \\
\hline $\begin{array}{l}\text { Thizade curclet } \\
\text { :tovil pit: }\end{array}$ & $-.10+0.28$ & $\tilde{z} .89 \pm 0.2 \mathrm{~K}$ & $367 \pm 0.58$ & $\therefore)=381$ & $0 . \ldots=6$ \\
\hline 3:1yt & 61500.22 & $5: 4: \pm 022$ & $3 \ll \pm 0 ! 4$ & S2 & $049:$ \\
\hline
\end{tabular}

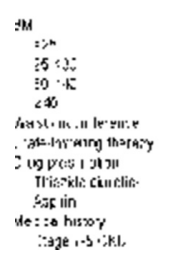

\begin{tabular}{|c|c|}
\hline 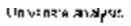 & 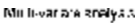 \\
\hline $1[:=x \tan t: z]$ & 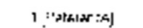 \\
\hline 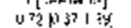 & $0=k+52)$ \\
\hline 0.48 p p.23 0.32y & $-00 k 112.5$ \\
\hline $0 A<\{0.22 .091]$ & i62|:6i-11.s \\
\hline 0.98 p..96.0 $\leqslant 9]$ & O \\
\hline $330[2.34 \cdot 4 \div 4]$ & $6 \mathrm{~m} \mid 3 / 4103:$ \\
\hline 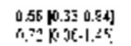 & 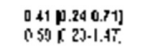 \\
\hline · & $0=3$ p.0y-0.ss] \\
\hline
\end{tabular}

Conclusions: The prevalence of gout in the US general population has not increased over the past 10 years. Although the use of urate lowering agents among patients with gout remained low, the percentage of patients with gout achieving the therapeutic target has increased over the past 10 years.

History of chronic kidney disease and use of thiazide diuretics are the most significant negative predictors for treatment success.

Disclosure of Interest: None declared

DOI: 10.1136/annrheumdis-2018-eular.5636

\section{FRI0252 TESTING FOR A CAUSAL ROLE OF MITOCHONDRIAL VARIATION IN THE DEVELOPMENT OF GOUT}

T.R. Merriman ${ }^{1}$, A. Shaukat ${ }^{1}$, A. Gosling ${ }^{1}$, M. Bixley ${ }^{1}$, N. Dalbeth ${ }^{2}$, L. Stamp ${ }^{3}$, E. Matisoo-Smith ${ }^{1}$, J. Harre Hindmarsh ${ }^{4}$, L. Joosten ${ }^{5}$, T. Jansen ${ }^{6}$, M. Janssen ${ }^{6}$, A. K. Tausche ${ }^{7}$, P. Riches ${ }^{8} .{ }^{1}$ University of Otago, Dunedin; ${ }^{2}$ University of Auckland, Auckland; ${ }^{3}$ University of Otago, Christchurch; ${ }^{4}$ Ngati Porou Hauora Charitable Trust, Te Puia Springs, New Zealand; ${ }^{5}$ Radboud University Medical Centre, Nijmegen; ${ }^{6}$ VieCuri Medical Centre, Venlo, Netherlands; ${ }^{7}$ University of Dresden, Dresden, Germany; ${ }^{8}$ University of Edinburgh, Edinburgh, UK

Background: Mitochondria execute critical roles in diverse cellular pathways. As a danger signal mitochondria induce inflammation in response to stress through NLRP3 inflammasome activation, central to gout development. We recently reported association of reduced mtDNA copy number $(\mathrm{CN})$ with prevalent gout in New Zealand Māori and Pacific (Polynesian) populations ${ }^{1}$. However the causeeffect relationship is unknown. This could be evaluated by testing for association with gout using nuclear genetic variants that associate with mtDNA CN.

Objectives: 1) Genome wide association study (GWAS) for mtDNA CN to identify nuclear and mitochondrial loci controlling mtDNA copy number 2) test any such loci for association with gout.

Methods: The mtDNA CN GWAS comprised 1340 Eastern Polynesian (EP), 816 Western Polynesian (WP) and 4579 European samples (New Zealand, Germany, The Netherlands, Scotland) genotyped on the Illumina CoreExome v24 array. 343 mitochondrial single nucleotide polymorphisms (SNPs) were evaluated. As previously described2 the median of the absolute difference in $\mathrm{X}$ and $\mathrm{Y}$ probe intensities was used as a measure of mtDNA CN, and additional 10000 randomly selected autosomal SNPs were used to calculate the principal components (PCs). A mtDNA CN GWAS was run on chromosomes 1-22 and the mitochondrial genome using Plink 1.9 .v2, adjusting for the first 10 PCs, age and sex followed by association analysis with gout adjusting by age, sex and the first $10 \mathrm{PCs}$ generated from a separate set of 3000 autosomal SNPs.

Results: The association of reduced mtDNA CN with gout in the EP and WP groups was reproduced but there was no evidence of association of mtDNA CN with gout in Europeans. Two genome-wide significant $(p<1 \times 10-7)$ variants MUC17 rs78010183 (T-allele) and SLC16A8 rs75640043 (T-allele) were associated with increased mitochondrial $\mathrm{CN}$ in EP and WP, respectively, and mitochon drial variant $r$ s3928306 was associated with mtDNA CN $\left(p=4.49 \times 10-1^{2}\right)$ in Europeans. MUC17 rs78010183 also associated with increased mtDNA CN in Europeans, with the $T$ allele also increasing $C N(\beta=0.06, p=1.07 \times 10-4)$. The $T$ allele of rs78010183 was associated with gout in Europeans (OR=9.32, $\mathrm{p}=5.53 \times 10-3)$ and the SLC16A8 $\mathrm{rs} 75640043 \mathrm{~T}$-allele was associated with gout in the WP group $(\mathrm{OR}=6.85, \mathrm{p}=5.50 \times 10-3)$. The mitochondrial variant $r s 3928306 \mathrm{~A}$ allele (very rare in Polynesian) was not associated with gout in Europeans $(\mathrm{OR}=1.09, \mathrm{p}=0.36)$.

Conclusions: That genetic variants associated with mitochondrial copy number also associate with gout provides evidence for a potential causal role of mitochondrial copy number in gout. However, the nuclear genetic variants support a causal relation of increased mtDNA CN with gout, conflicting with our previous observational report of association of reduced mtDNA CN with gout ${ }^{1}$.

\section{REFERENCES:}

[1] Gosling A, et al. Mitochondrial genetic variation and gout in Māori and Pacific people living in Aotearoa New Zealand. Ann Rheum Dis 2017 Dec 15. [Epub ahead of print].

[2] Asher FN, et al. Association of mitochondrial DNA levels with frailty and all-cause mortality. J Mol Med 2015;93:177-86.

Disclosure of Interest: None declared

DOI: 10.1136/annrheumdis-2018-eular.5017

\section{FRI0253 1 TRENDS FOR GOUT IN ADULTS IN AN URBAN AREA FOR A 5-YEAR PERIOD: INCIDENCE, PREVALENCE AND HOSPITALISATION RATES}

V. Dostanko $^{1}$, V. Yagur ${ }^{1}$, V. Apanasovich ${ }^{1}$, N. Dostanko ${ }^{1}$, A. Rekun ${ }^{2} .{ }^{1} 2-d$ Department of Internal Medicine, BSMU; ${ }^{2}$ Rheumatology Department, 9-th Minsk City Clinical Hospital, Minsk, Belarus

Background: Gout is one of the most common arthritis nowadays which has a great influence on patient's quality of life, course and outcomes of cardiovascular and renal pathology. ${ }^{1}$ Some recent papers demonstrate that the prevalence of gout has risen over the last decades and underline the scarcity or lack of epidemiologic data in different countries as well as the notable variations among them.

Objectives: We estimated incidence, prevalence and hospitalisation rates for gout in Minsk (the Republic of Belarus) for the 5 year period (2011-2015).

Methods: Minsk is a typical urban area which is considered to be representative for the urban population of the whole country. The data on the new onset gout and the first visit for gout in a corresponding year were collected from all rheumatologic services of Minsk for the 5 year period from January, 1 of 2011 to December, 31 of 2015. Only patients older than 18 years old with the diagnosis of gout according to the ICD-10 (M10) were included. The data on age and sex structure of the population of the Republic of Belarus were obtained from the annual Statistical bulletins of the National Statistical Committee of the Republic of Belarus. Hospitalisation rates were calculated on the base of statistical reports on discharges for the corresponding year.

Results: There were no significant differences in age and sex structure of the population in Belarus and Minsk for the study period. The size of the adult population, incidence and prevalence of gout, hospitalisation rates and duration of hospitalizations for gout for the corresponding years in Minsk are presented in the table 1. According to these data the gout incidence for the 5 year period changed from 25.0 (Cl 95\% 24.7-25.2) to 35.5 (Cl 95\% 35.3-35.8) per 100000 adults, the gout prevalence for the period 2011-2015 also increased from $178.9(\mathrm{Cl} 95 \% 178.3$ 179.5) to 290.3 ( $\mathrm{Cl} 95 \% 289.6-291.0)$ per 100000 adults $(p<0.001$ for both trends). There were 2688 hospitalizations in Minsk for gout for the study period. The mean age of hospitalised patients was $57.3 \pm 10.3$ years (median 58.0; range $25-87), 91.4 \%$ were men $(92 \% ; 87.8 \%-94.9 \%)$. It is worth noting that $45.6 \%$ of hospitalised patients were at the age $55-65 \%$ and $90.2 \%$ were under 70 years old with sharp decrease of hospitalizations after 65 years of age. Hospitalisation rates 